\title{
Bifurcation and Nodal Solutions for the Half-Linear Problems with Nonasymptotic Nonlinearity at 0 and $\infty$
}

\author{
Wenguo Shen \\ Department of Basic Courses, Lanzhou Institute of Technology, Lanzhou 730050, China \\ Correspondence should be addressed to Wenguo Shen; shenwg369@163.com
}

Received 26 May 2015; Accepted 17 August 2015

Academic Editor: Gabriele Bonanno

Copyright (C) 2016 Wenguo Shen. This is an open access article distributed under the Creative Commons Attribution License, which permits unrestricted use, distribution, and reproduction in any medium, provided the original work is properly cited.

We study the existence of nodal solutions for the following problem: $-x^{\prime \prime}=\alpha x^{+}+\beta x^{-}+r a(t) f(x), 0<t<1, x(0)=x(1)=0$, where $r \neq 0$ is a parameter, $a(t) \in C([0,1],(0, \infty))$ with $a(t) \neq \equiv \quad 0$ on any subinterval of $[0,1], x^{+}=\max \{x, 0\}, x^{-}=-\min \{x, 0\}$, and $\alpha, \beta \in C[0,1] ; f \in C(\mathbb{R}, \mathbb{R}), s f(s)>0$ for $s \neq 0$, and $f_{0}, f_{\infty} \notin(0, \infty)$, where $f_{0}=\lim _{|s| \rightarrow 0} f(s) / s$ and $f_{\infty}=\lim _{|s| \rightarrow+\infty} f(s) / s$. We use bifurcation techniques to prove our main results.

\section{Introduction}

By applying the bifurcation techniques of Rabinowitz $[1,2]$, Im et al. [3] studied the existence of positive solutions of the problems and some authors [4-7] studied the existence of nodal solutions of the problems. Recently, Dai and Ma [8] established the unilateral global bifurcation theory for the problems by applying the bifurcation techniques of Dancer [9]. Later, Dai [10] also considered the existence of nodal solutions for the problems with nonasymptotic nonlinearity at 0 or $\infty$ by applying the unilateral global bifurcation theory of [8]. For the abstract unilateral global bifurcation theory, we refer the reader to $[1,2,8,9,11-13]$ and the references therein. On the other hand, problems involving nondifferentiable nonlinearity have also been investigated by applying bifurcation techniques; see [13-17]. In particular, Berestycki [14] established the Rabinowitz-type global interval bifurcation result and Ma and Dai [13] established the unilateral global interval bifurcation theorem. Meanwhile, half-linear or halfquasilinear boundary value problems have attracted the attention of many specialists in different equations because of their interesting applications; see [13, 14, 16-18]. Among them, Berestycki [14] (or see [13]) established the spectrum for the following half-linear eigenvalue problem:

$$
\begin{aligned}
& -x^{\prime \prime}=\lambda a(t) x+\alpha x^{+}+\beta x^{-}, \quad 0<t<1, \\
& x(0)=x(1)=0,
\end{aligned}
$$

where $x^{+}=\max \{x, 0\}, x^{-}=-\min \{x, 0\}, \alpha, \beta \in C[0,1]$, and $a$ satisfies the following condition:

(A1) $a \in C([0,1],(0, \infty))$ with $a(t) \not \equiv 0$ on any subinterval of $[0,1]$.

Let $Y=C[0,1]$ with the norm $\|x\|_{\infty}=\max _{t \in[0,1]}|x(t)|$. Let $E=\left\{x \in C^{1}[0,1] \mid x(0)=x(1)=0\right\}$ with the norm $\|x\|=\max \left\{\|x\|_{\infty},\left\|x^{\prime}\right\|_{\infty}\right\}$.

We define the linear operator $L: D(L) \subset E \rightarrow Y$

$$
L x=-x^{\prime \prime}, \quad x \in D(L)
$$

with $D(L)=\left\{x \in C^{2}[0,1] \mid x(0)=x(1)=0\right\}$. Then $L$ is a closed operator and $L^{-1}: Y \rightarrow E$ is completely continuous.

Let $\mathbf{E}=\mathbb{R} \times E$ under the product topology. Let $S_{k}^{+}$denote the set of function in $E$ which have exactly $k-1$ interior nodal (i.e., nondegenerate) zeros in $(0,1)$ and are positive near $t=0$, set $S_{k}^{-}=-S_{k}^{+}$, and $S_{k}=S_{k}^{+} \cup S_{k}^{-}$. They are disjoint and open in $E$. Finally, let $\Phi_{k}^{ \pm}=\mathbb{R} \times S_{k}^{ \pm}$and $\Phi_{k}=\mathbb{R} \times S_{k}$.

By Theorem 1 of [14], Berestycki [14] obtained the following result.

Lemma 1 (see [14, Theorem 2]). Let (A1) hold. There exist two sequences of simple half eigenvalues for (1)

$$
\begin{aligned}
& \lim _{k \rightarrow \infty} \lambda_{k}^{+}=+\infty, \quad \lambda_{1}^{+}<\lambda_{2}^{+}<\cdots<\lambda_{k}^{+}<\cdots, \\
& \lim _{k \rightarrow \infty} \lambda_{k}^{-}=+\infty, \quad \lambda_{1}^{-}<\lambda_{2}^{-}<\cdots<\lambda_{k}^{-}<\cdots .
\end{aligned}
$$


The corresponding half-lines solutions are in $\left\{\lambda_{k}^{+}\right\} \times S_{k}^{+}$and $\left\{\lambda_{k}^{-}\right\} \times S_{k}^{-}$. Further, aside from these solutions and the trivial ones, there are no other solutions of (1).

Furthermore, following Lemma 1, Ma and Dai [13] (or see [14]) considered the existence of nodal solutions for the following half-linear eigenvalue problem:

$$
\begin{aligned}
& -x^{\prime \prime}=\alpha x^{+}+\beta x^{-}+r a(t) f(x), \quad 0<t<1, \\
& x(0)=x(1)=0,
\end{aligned}
$$

where $r \neq 0$ is a parameter and $f \in C(\mathbb{R}, \mathbb{R})$ satisfies the following assumptions:

(A2) $s f(s)>0$ for $s \neq 0$.

(A3) There exist $f_{0}, f_{\infty} \in(0,+\infty)$ such that

$$
\begin{aligned}
f_{0} & =\lim _{|s| \rightarrow 0} \frac{f(s)}{s}, \\
f_{\infty} & =\lim _{|s| \rightarrow+\infty} \frac{f(s)}{s} .
\end{aligned}
$$

Firstly, we show that the existence and uniqueness theorem is valid for (4).

Lemma 2 (see [13, Lemma 2.2]). If $(\lambda, x)$ is a nontrivial solution of (4) under assumptions $(A 1)-(A 3)$ and $x$ has a double zero, then $x \equiv 0$.

Under assumptions $(A 1)-(A 3)$, they obtained the following result.

Theorem 3 (see [13, Theorem 4.1]). Let (A1), (A2), and (A3) hold. For $v \in\{+,-\}$, either $\lambda^{v} / f_{\infty}<r<\lambda^{v} / f_{0}$ or $\lambda^{v} / f_{0}<$ $r<\lambda^{v} / f_{\infty}$. Then problem (4) has a solution $x_{k}^{v}$ such that $x_{k}^{v}$ has exactly $k-1$ zeros in $(0,1)$ and $\nu x_{k}^{v}$ is positive near 0 .

Of course, the natural question is that of what would happen if $f_{0} \notin(0,+\infty)$ or $f_{\infty} \notin(0,+\infty)$. Obviously, the previous results cannot deal with this case. The purpose of this work is to establish several results similar to those of [13]. The main methods used in this work are global bifurcation techniques and the approximation of connected components. Moreover, we consider the cases of $f_{0}, f_{\infty} \notin(0, \infty)$, while the authors of $[13,16,17]$ only studied the cases of $f_{0}, f_{\infty} \in$ $(0, \infty)$.

In this paper, we will investigate the existence of nodal solutions for problem (4), where $a$ satisfies condition $(A 1)$. Throughout this paper, we assume that $f$ satisfies $(A 2)$ and the following assumptions:

(H1) $f_{0} \in(0, \infty)$ and $f_{\infty}=0$.

(H2) $f_{0} \in(0, \infty)$ and $f_{\infty}=\infty$.

(H3) $f_{0}=\infty$ and $f_{\infty} \in(0, \infty)$.

(H4) $f_{0}=\infty$ and $f_{\infty}=0$.

(H5) $f_{0}=\infty$ and $f_{\infty}=\infty$.

(H6) $f_{0}=0$ and $f_{\infty} \in(0, \infty)$.
(H7) $f_{0}=0$ and $f_{\infty}=0$.

(H8) $f_{0}=0$ and $f_{\infty}=\infty$.

The rest of this paper is arranged as follows. In Section 2, we gave some preliminaries. In Section 3, we give the interval for the parameter $r$ which ensure the existence of single or multiple nodal solutions for half-linear problem (4) under assumptions (A2) and (H1)-(H8) for the nonlinearity $f$.

\section{Preliminaries}

Let $\delta_{k}^{v}$ denote the closure of the set of nontrivial solutions of (4) in $\mathbb{R} \times E$, with $\mathcal{S}_{k}^{v}$ denoting the subset of with $u \in S_{k}^{ \pm}$and $\delta_{k}=\mathcal{S}_{k}^{+} \cup \mathcal{S}_{k}^{-}$.

In order to prove our main results, we need the following Sturm type comparison result.

Lemma 4. Let $b_{2}(t) \geq \max \left\{b_{1}(t), b_{1}(t)+\alpha(t)+\beta(t), b_{1}(t)-\right.$ $\alpha(t)-\beta(t)\}$ for $t \in(0,1)$ and $b_{i}(t) \in C(0,1), i=1,2$. Also let $u_{1}, u_{2}$ be solutions of the following differential equations:

$$
u^{\prime \prime}+b_{i}(t) u+\alpha u^{+}+\beta u^{-}=0, \quad t \in(0,1), i=1,2
$$

respectively. If $(c, d) \subseteq(0,1)$, and $u_{1}(c)=u_{1}(d)=0, u_{1}(t) \neq 0$ in $(c, d)$, then either there exists $\tau \in(c, d)$ such that $u_{2}(\tau)=0$ or $b_{2}=b_{1}$ or $b_{2}=b_{1}+\alpha+\beta$ or $b_{2}=b_{1}-\alpha-\beta$ and $u_{2}(t)=\mu u_{1}(t)$ for some constant $\mu \neq 0$.

Proof. We discuss four cases.

Case $1\left(u_{1}(t)>0, u_{2}(t)>0\right.$ in $\left.(c, d)\right)$. By the Picone identity [19] (or (0.4) of [20]), we have

$$
\begin{gathered}
\int_{c}^{d}\left[\frac{u_{1}}{u_{2}}\left(u_{2} u_{1}^{\prime}-u_{1} u_{2}^{\prime}\right)\right] d t \\
=\int_{c}^{d} \frac{u_{1}}{u_{2}}\left(u_{2} \widehat{L} u_{1}-u_{1} \widehat{L} u_{2}\right) d t \\
+\int_{c}^{d}\left(u_{1}^{\prime}-\frac{u_{1}}{u_{2}} u_{2}^{\prime}\right)^{2} d t,
\end{gathered}
$$

where $\widehat{L} x=-L x=x^{\prime \prime}, x \in D(L)$. It follows that

$$
\begin{aligned}
& \int_{c}^{d}\left[\frac{u_{1}}{u_{2}}\left(u_{2} u_{1}^{\prime}-u_{1} u_{2}^{\prime}\right)\right] d t \\
& \quad=\int_{c}^{d}\left[\left(b_{2}-b_{1}\right) u_{1}^{2}+\left(u_{1}^{\prime}-\frac{u_{1}}{u_{2}} u_{2}^{\prime}\right)^{2}\right] d t .
\end{aligned}
$$

The left-hand side of (8) equals

$$
\begin{aligned}
& -\lim _{t \rightarrow c^{+}} \frac{u_{1}\left(u_{2} u_{1}^{\prime}-u_{1} u_{2}^{\prime}\right)}{u_{2}}+\lim _{t \rightarrow d^{-}} \frac{u_{1}\left(u_{2} u_{1}^{\prime}-u_{1} u_{2}^{\prime}\right)}{u_{2}} \\
& \quad=-H_{c}+H_{d} .
\end{aligned}
$$


We prove that $H_{c}=0$. If $u_{2}(c) \neq 0$, then $H_{c}=0$. If $u_{2}(c)=0$, noting the conclusion of Lemma 2 , then $u_{2}^{\prime}(c)>0$. By L'Hospital rule, we have that

$$
\begin{aligned}
H_{c} & =\lim _{t \rightarrow c^{+}} \frac{u_{1}\left(u_{2} u_{1}^{\prime}-u_{1} u_{2}^{\prime}\right)}{u_{2}} \\
& =\lim _{t \rightarrow c^{+}} \frac{u_{1}^{\prime}\left(u_{2} u_{1}^{\prime}-u_{1} u_{2}^{\prime}\right)+u_{1}\left(u_{2} u_{1}^{\prime}-u_{1} u_{2}^{\prime}\right)^{\prime}}{u_{2}^{\prime}}=0 .
\end{aligned}
$$

Similarly, we can obtain that $H_{d}=0$. Therefore, the left-hand side of (8) equals zero. Hence, the right-hand side of (8) also equals zero. It follows that there exists a constant $\mu \neq 0$ such that $u_{2}=\mu u_{1}$ and $b_{2}=b_{1}$.

Case $2\left(u_{1}(t)>0, u_{2}(t)<0\right.$ in $\left.(c, d)\right)$. Similar to (8), we can get

$$
\begin{aligned}
& \int_{c}^{d}\left[\frac{u_{1}}{u_{2}}\left(u_{2} u_{1}^{\prime}-u_{1} u_{2}^{\prime}\right)\right] d t \\
& \quad=\int_{c}^{d}\left[\left(b_{2}-b_{1}-\alpha-\beta\right) u_{1}^{2}+\left(u_{1}^{\prime}-\frac{u_{1}}{u_{2}} u_{2}^{\prime}\right)^{2}\right] d t .
\end{aligned}
$$

The above argument is still valid for this case.

Case $3\left(u_{1}(t)<0, u_{2}(t)<0\right.$ in $\left.(c, d)\right)$. Similar to Case 1 , we can get the result.

Case $4\left(u_{1}(t)<0, u_{2}(t)>0\right.$ in $\left.(c, d)\right)$. Similar to (8), we can get

$$
\begin{aligned}
& \int_{c}^{d}\left[\frac{u_{1}}{u_{2}}\left(u_{2} u_{1}^{\prime}-u_{1} u_{2}^{\prime}\right)\right]^{\prime} d t \\
& \quad=\int_{c}^{d}\left[\left(b_{2}-b_{1}+\alpha+\beta\right) u_{1}^{2}+\left(u_{1}^{\prime}-\frac{u_{1}}{u_{2}} u_{2}^{\prime}\right)^{2}\right] d t .
\end{aligned}
$$

Similar to the proof of Case 1, we can obtain the result.

By Lemma 4, we obtain the following result that will be used later.

Lemma 5. Let $I=[a, b]$ be such that $I \subset[0,1]$ and

$$
\text { meas }(I)>0 \text {. }
$$

Let $g_{n}(t) \in C([0,1], \mathbb{R})$ be such that

$$
\lim _{n \rightarrow+\infty} g_{n}(t)=+\infty \quad \text { uniformly on } I \text {. }
$$

Let $y_{n} \in E$ be a solution of the equation

$$
y_{n}^{\prime \prime}(t)+g_{n}(t) y_{n}+\alpha y_{n}^{+}+\beta y_{n}^{-}=0, \quad 0<t<1 .
$$

Then $y_{n}$ must change sign on $I$ as $n \rightarrow+\infty$.
Proof. Set $\alpha^{0}:=\max _{t \in[0,1]}|\alpha(t)|$ and $\beta^{0}:=\max _{t \in[0,1]}|\beta(t)|$. By simple computation, we can show that

$$
g_{n}(t)+\alpha \frac{y_{n}^{+}}{y_{n}}+\beta \frac{y_{n}^{-}}{y_{n}} \geq g_{n}(t)-\alpha^{0}-\beta^{0} \quad \forall t \in(0,1) .
$$

After taking a subsequence if necessary, we may assume that

$$
g_{n}(t)-\alpha^{0}-\beta^{0} \geq \lambda_{j} \quad \forall t \in I
$$

as $j \rightarrow+\infty$, where $\lambda_{j}$ is $j$ th eigenvalue of the following problem:

$$
\begin{gathered}
u^{\prime \prime}+\lambda u=0, \quad 0<t<1, \\
u(0)=u(1)=0 .
\end{gathered}
$$

Set $s=(b-a) t+a, u(s)=v((b-a) s+a)$. By some simple computations, we can show

$$
\begin{aligned}
(b-a)^{2} v^{\prime \prime}+\lambda v & =0, \quad t \in(a, b), \\
v(a) & =v(b)=0 .
\end{aligned}
$$

Let $\psi_{j}$ be the corresponding eigenfunction of $\lambda_{j}$. Since $\lambda_{j} \rightarrow$ $\infty$ as $j \rightarrow \infty$, Lemma 2.1 of [4] implies that $\psi_{j}$ must change sign on $I$. Note that the conclusion of Lemma 4 also is valid if $\alpha=\beta=0$. Using these facts and Lemma 4 , we can obtain the desired result.

Definition 6 (see [21]). Let $X$ be a Banach space and $\left\{C_{n} \mid n=\right.$ $1,2, \ldots\}$ be a family of subsets of $X$. Then the superior limit $\mathbb{D}$ of $\left\{C_{n}\right\}$ is defined by

$$
\begin{aligned}
\mathbb{D} D & :=\limsup _{n \rightarrow \infty} C_{n}=\left\{x \in X \mid \exists\left\{n_{i}\right\} \subset \mathbb{N}, x_{n_{i}}\right. \\
& \left.\in C_{n_{i}} \text {, such that } x_{n_{i}} \longrightarrow x\right\} .
\end{aligned}
$$

Lemma 7 (see [22]). Let $X$ be a Banach space and let $\left\{C_{n} \mid n=\right.$ $1,2, \ldots\}$ be a family of closed connected subsets of $X$. Assume that

(i) there exist $z_{n} \in C_{n}, n=1,2, \ldots$, and $z^{*} \in X$, such that $z_{n} \rightarrow z^{*}$

(ii) $r_{n}=\sup \left\{\|x\| \mid x \in C_{n}\right\}=\infty$;

(iii) for all $R>0,\left(\bigcup_{n=1}^{\infty} C_{n}\right) \cap B_{R}$ is a relative compact set of $X$, where

$$
B_{R}=\{x \in X \mid\|x\| \leq R\} .
$$

Then there exists an unbounded component $C$ in $\mathbb{D}$ and $z^{*} \in C$.

\section{Nodal Solutions for Half-Linear Eigenvalue Problems}

In this section, we will study the existence of nodal solutions for problem (4) with $f_{0} \notin(0,+\infty)$ or $f_{\infty} \notin(0,+\infty)$.

We start this section by studying the following eigenvalue problem:

$$
\begin{aligned}
& -x^{\prime \prime}=\alpha x^{+}+\beta x^{-}+\lambda r a(t) f(x), \quad 0<t<1, \\
& x(0)=x(1)=0,
\end{aligned}
$$


where $\lambda>0$ is a parameter. Firstly, under the conditions $(A 1)-(A 3)$, let $\zeta \in C(\mathbb{R}, \mathbb{R})$ be such that

$$
f(x)=f_{0} x+\zeta(x)
$$

with $\lim _{|x| \rightarrow 0}(\zeta(x) / x)=0$. Let us consider

$$
\begin{aligned}
&-x^{\prime \prime}=\lambda r a(t) f_{0} x+\alpha x^{+}+\beta x^{-}+\lambda r a(t) \zeta(x), \\
& 0<t<1,
\end{aligned}
$$

$$
x(0)=x(1)=0,
$$

as a bifurcation problem from the trivial solution $x \equiv 0$. By using Theorem 3 of [13] or Ma and Dai [13, Lemma 4.1] obtained the following Lemma.

Lemma 8 (see [13, Lemma 4.1]). For $v \in\{+,-\},\left(\lambda_{k}^{v} / r f_{0}, 0\right)$ is a bifurcation point for problem (24). Moreover, there exists an unbounded continuum $\mathscr{D}_{k}^{v}$ of solutions of problem (24), such that $\mathscr{D}_{k}^{\nu} \subset\left(\Phi_{k}^{v} \cup\left\{\left(\lambda_{k}^{v} / r f_{0}, 0\right)\right\}\right)$.

Remark 9. Any solution of $(22)$ of the form $(1, x)$ yields a solution $x$ of (4). In order to prove our main results, one will only show that $\mathscr{D}_{k}^{v}$ crosses the hyperplane $\{1\} \times E$ in $\mathbb{R} \times E$.

Clearly, (A2) implies $f(0)=0$. Hence, $x=0$ is always the solution of (4). Applying Lemma 8 (or Lemma 4.1 of [13]), we will establish the existence of nodal solutions of (4) as follows.

Theorem 10. Let (A1), (A2), and (H1) hold. For $v \in\{+,-\}$, assume that one of the following conditions holds:

(i) $r \in\left(\lambda_{k}^{v} / f_{0},+\infty\right)$ for $\lambda_{k}^{v}>0$.

(ii) $r \in\left(-\infty, \lambda_{k}^{-} / f_{0}\right) \cup\left(\lambda_{k}^{+} / f_{0},+\infty\right)$ for $\nu \lambda_{k}^{v}>0$.

(iii) $r \in\left(-\infty, \lambda_{k}^{+} / f_{0}\right) \cup\left(\lambda_{k}^{-} / f_{0},+\infty\right)$ for $\nu \lambda_{k}^{v}<0$.

(iv) $r \in\left(-\infty, \lambda_{k}^{v} / f_{0}\right)$ for $\lambda_{k}^{v}<0$.

Then problem (4) possesses two solutions $x_{k}^{+}$and $x_{k}^{-}$such that $x_{k}^{+}$has exactly $k-1$ zeros in $(0,1)$ and is positive near 0 and $x_{k}^{-}$has exactly $k-1$ zeros in $(0,1)$ and is negative near 0 .

Proof. We only prove the case of (i) since the proofs of the cases for (ii), (iii), and (iv) can be given similarly.

In view of the proof to prove [13, Theorem 4.1], we only need to show that $\mathscr{D}_{k}^{v}$ joins $\left(\lambda_{k}^{v} / r f_{0}, 0\right)$ to $(\infty, \infty)$. To do this, it is enough to prove that $\left[\lambda_{k}^{v} / r f_{0},+\infty\right) \subset \operatorname{Proj}_{R} \mathscr{D}_{k}^{v}$.

Assume on the contrary that $\sup \left\{\lambda \mid(\lambda, u) \in \mathscr{D}_{k}^{\nu}\right\}<+\infty$; then there exists a sequence $\left(\mu_{n}, x_{n}\right) \in \mathscr{D}_{k}^{v}$ such that

$$
\begin{gathered}
\lim _{n \rightarrow \infty}\left\|x_{n}\right\|=+\infty, \\
\mu_{n} \leq c_{0},
\end{gathered}
$$

for some positive constant $c_{0}$ not depending on $n$.

By $(H 1)$, let $\bar{f}(x)=\max _{0 \leq|s| \leq x}|f(s)|$, then $\bar{f}$ is nondecreasing and

$$
\lim _{x \rightarrow+\infty} \frac{\bar{f}(x)}{x}=0
$$

We consider the equation

$$
\begin{aligned}
-x_{n}^{\prime \prime} & =\alpha x_{n}^{+}+\beta x_{n}^{-}+\mu_{n} r a(t) f\left(x_{n}\right), \quad t \in(0,1), \\
x_{n}(0) & =x_{n}(1)=0 .
\end{aligned}
$$

Let $y_{n}=x_{n} /\left\|x_{n}\right\|$, and $y_{n}$ should be the solutions of problem

$$
\begin{aligned}
& -y_{n}^{\prime \prime}=\alpha y_{n}^{+}+\beta y_{n}^{-}+\mu_{n} r a(t) \frac{f\left(x_{n}\right)}{\left\|x_{n}\right\|}, \quad t \in(0,1), \\
& y_{n}(0)=y_{n}(1)=0 .
\end{aligned}
$$

Since $y_{n}$ is bounded in $C^{2}[0,1]$, choosing a subsequence and relabelling if necessary, we have that $y_{n} \rightarrow y$ for some $y \in E$ and $\|y\|=1$.

Furthermore, from (26) and the fact that $\bar{f}$ is nondecreasing, we have that

$$
\lim _{n \rightarrow \infty} \frac{f\left(x_{n}\right)}{\left\|x_{n}\right\|}=0,
$$

since

$$
\begin{aligned}
\frac{f\left(x_{n}\right)}{\left\|x_{n}\right\|} \leq \frac{\bar{f}\left(\left|x_{n}\right|\right)}{\left\|x_{n}\right\|} \leq \frac{\bar{f}\left(\left\|x_{n}\right\|_{\infty}\right)}{\left\|x_{n}\right\|} \leq \frac{\bar{f}\left(\left\|x_{n}\right\|\right)}{\left\|x_{n}\right\|} & \longrightarrow 0, \\
n & \longrightarrow+\infty .
\end{aligned}
$$

By (29) and the compactness of $L^{-1}$, we obtain that

$$
\begin{aligned}
& -y^{\prime \prime}=\alpha y^{+}+\beta y^{-}, \quad t \in(0,1), \\
& y(0)=y(1)=0 .
\end{aligned}
$$

By $y(0)=y(1)$, there exists $\xi \in(0,1)$ such that $y^{\prime}(\xi)=0$. Without loss of generality, it follows that

$$
\begin{array}{r}
|y(t)|=\left|\int_{0}^{t}\left[\int_{\xi}^{s}\left(-\alpha y^{+}-\beta y^{-}\right) d \tau\right] d s\right| \leq M \int_{0}^{t}|y| d s, \\
t \in(0,1),
\end{array}
$$

$M=\max \left\{\alpha^{0}, \beta^{0}\right\}$.

By the Gronwall-Bellman inequality [3, Lemma 2.1], we get $y(t) \equiv 0, \forall t \in[0,1]$.

This contradicts $\|y(t)\|=1$.

Theorem 11. Let (A1), (A2), and (H2) hold. For $v \in\{+,-\}$, assume that one of the following conditions holds:

(i) $r \in\left(0, \lambda_{k}^{v} / f_{0}\right)$ for $\lambda_{k}^{v}>0$.

(ii) $r \in\left(0, \lambda_{k}^{+} / f_{0}\right) \cup\left(\lambda_{k}^{-} / f_{0}, 0\right)$ for $\nu \lambda_{k}^{v}>0$.

(iii) $r \in\left(0, \lambda_{k}^{-} / f_{0}\right) \cup\left(\lambda_{k}^{+} / f_{0}, 0\right)$ for $\nu \lambda_{k}^{v}<0$.

(iv) $r \in\left(\lambda_{k}^{v} / f_{0}, 0\right)$ for $\lambda_{k}^{v}<0$.

Then problem (4) possesses two solutions $x_{k}^{+}$and $x_{k}^{-}$such that $x_{k}^{+}$has exactly $k-1$ zeros in $(0,1)$ and is positive near 0 and $x_{k}^{-}$has exactly $k-1$ zeros in $(0,1)$ and is negative near 0 .

Proof. We will only prove the case of (i) since the proofs of the cases for (ii), (iii), and (iv) are completely analogous. 
Inspired by the idea of [23] or see [10], we define the cut-off function of $f$ as follows:

$$
f^{[n]}(s):= \begin{cases}f(s), & s \in[-n, n], \\ \frac{2 n^{2}-f(n)}{n}(s-n)+f(n), & s \in(n, 2 n), \\ \frac{2 n^{2}+f(-n)}{n}(s+n)+f(-n), & s \in(-2 n,-n), \\ n s, & s \in(-\infty,-2 n] \cup[2 n,+\infty) .\end{cases}
$$

We consider the following problem:

$$
\begin{aligned}
& -x^{\prime \prime}=\alpha x^{+}+\beta x^{-}+\lambda r a(t) f^{[n]}(x), \quad 0<t<1, \\
& x(0)=x(1)=0 .
\end{aligned}
$$

Clearly, we can see that $\lim _{n \rightarrow+\infty} f^{[n]}(s)=f(s),\left(f^{[n]}\right)_{0}=$ $f_{0}$, and $\left(f^{[n]}\right)_{\infty}=n$.

Similar to the proof of [13, Theorem 4.1], by Lemma 8 and Remark 9, there exists an unbounded continuum $\mathscr{D}_{k}^{\nu[n]}$ of solutions of problem (34) emanating from $\left(\lambda_{k}^{v} / r f_{0}, 0\right)$, such that $\mathscr{D}_{k}^{v[n]} \subset\left(\Phi_{k}^{v} \cup\left\{\left(\lambda_{k}^{v} / r f_{0}, 0\right)\right\}\right)$, and $\mathscr{D}_{k}^{v[n]}$ joins $\left(\lambda_{k}^{v} / r f_{0}, 0\right)$ to $\left(\lambda_{k}^{v} / r n, \infty\right)$.

Taking $z_{n}=\left(\lambda_{k}^{v} / r n, \infty\right)$ and $z^{*}=(0, \infty)$, we have that $z_{n} \rightarrow z^{*}$.

So condition (i) in Lemma 7 is satisfied with $z^{*}=(0, \infty)$.

Obviously

$$
r_{n}=\sup \left\{\lambda+\|x\| \mid(\lambda, x) \in \mathscr{D}^{\nu[n]}\right\}=\infty,
$$

and, accordingly (ii) in Lemma 7 holds. (iii) in Lemma 7 can be deduced directly from the Arzela-Ascoli Theorem and the definition of $f^{[n]}$.

Therefore, by Lemma 7, $\lim \sup _{n \rightarrow \infty} \mathscr{D}_{k}^{v[n]}$ contains an unbounded connected component $\mathscr{D}_{k}^{\nu}$ with $(0, \infty) \in \mathscr{D}_{k}^{\nu}$.

From $\lim _{n \rightarrow+\infty} f^{[n]}(s)=f(s),(34)$ can be converted to equivalent equation (22). Since $\mathscr{D}_{k}^{v[n]} \subset \Phi_{k}^{v}$, we conclude $\mathscr{D}_{k}^{v} \subset \Phi_{k}^{v}$. Moreover, $\mathscr{D}_{k}^{v} \subset \mathcal{S}_{k}^{v}$ by (4).

In the following, we show that $\left(\lambda_{k}^{v} / r f_{0}, 0\right) \in \mathscr{D}_{k}^{v}$.

Let $\left(\mu_{n}, x_{n}\right) \in \mathscr{D}_{k}^{v} \backslash\left\{\left(\lambda_{k}^{v} / r f_{0}, 0\right)\right\}$ satisfy $\lim _{n \rightarrow \infty}\left\|x_{n}\right\|=0$.

Let $\zeta \in C(\mathbb{R}, \mathbb{R})$ be such that

$$
f(s)=f_{0} s+\zeta(s) .
$$

With $\lim _{|s| \rightarrow 0}(\zeta(s) / s)=0$, let $\bar{\zeta}(x)=\max _{0 \leq|s| \leq x}|\zeta(s)|$, then $\bar{\zeta}$ is nondecreasing and

$$
\lim _{x \rightarrow \infty} \frac{\bar{\zeta}(x)}{x}=0 .
$$

We divide the equation

$$
\begin{array}{ll}
-x_{n}^{\prime \prime}=\mu_{n} r a(t) f_{0} x_{n}+\alpha x_{n}^{+}+\beta x_{n}^{-}+\lambda r a(t) \zeta\left(x_{n}\right), & 0<t<1, \\
x_{n}(0)=x_{n}(1)=0 . &
\end{array}
$$

Let $y_{n}=x_{n} /\left\|x_{n}\right\|$, and $y_{n}$ should be the solutions of problem

$$
\begin{array}{r}
-y_{n}^{\prime \prime}=\mu_{n} r a(t) f_{0} y_{n}+\alpha y_{n}^{+}+\beta y_{n}^{-}+\mu_{n} r a(t) \frac{\zeta\left(x_{n}\right)}{\left\|x_{n}\right\|}, \\
t \in(0,1),
\end{array}
$$

$$
y_{n}(0)=y_{n}(1)=0 \text {. }
$$

Since $y_{n}$ is bounded in $C^{2}[0,1]$, choosing a subsequence and relabelling if necessary, we have that $y_{n} \rightarrow y$ for some $y \in E$.

Furthermore, from (37) and the fact that $\bar{\zeta}$ is nondecreasing, we have that

$$
\lim _{n \rightarrow \infty} \frac{\zeta\left(x_{n}\right)}{\left\|x_{n}\right\|}=0
$$

since

$$
\begin{aligned}
& \frac{\zeta\left(x_{n}\right)}{\left\|x_{n}\right\|} \leq \frac{\bar{\zeta}\left(\left|x_{n}\right|\right)}{\left\|x_{n}\right\|} \leq \frac{\bar{\zeta}\left(\left\|x_{n}\right\|_{\infty}\right)}{\left\|x_{n}\right\|} \leq \frac{\bar{\zeta}\left(\left\|x_{n}\right\|\right)}{\left\|x_{n}\right\|} \longrightarrow 0, \\
& n \longrightarrow+\infty \text {. }
\end{aligned}
$$

By (40) and the compactness of $L^{-1}$, we obtain that

$$
L y=\mu r a(t) f_{0} y+\alpha y^{+}+\beta y^{-} .
$$

It is clear that $\|y\|=1$ and $y \in \overline{\mathscr{D}_{k}^{v}} \subseteq \mathscr{D}_{k}^{v}$ since $\mathscr{D}_{k}^{v}$ is closed in $\mathbb{R} \times E$. Moreover, by Lemma $1, \mu r f_{0}=\lambda_{k}^{v}$, so that

$$
\mu=\frac{\lambda_{k}^{v}}{r f_{0}} .
$$

Thus $\mathscr{D}_{k}^{v}$ joins $\left(\lambda_{k}^{v} / r f_{0}, 0\right)$ to $(0, \infty)$.

Theorem 12. Let (A1), (A2), and (H3) hold. For $v \in\{+,-\}$, assume that one of the following conditions holds:

(i) $r \in\left(0, \lambda_{k}^{v} / f_{\infty}\right)$ for $\lambda_{k}^{v}>0$.

(ii) $r \in\left(0, \lambda_{k}^{+} / f_{\infty}\right) \cup\left(\lambda_{k}^{-} / f_{\infty}, 0\right)$ for $v \lambda_{k}^{v}>0$.

(iii) $r \in\left(0, \lambda_{k}^{-} / f_{\infty}\right) \cup\left(\lambda_{k}^{+} / f_{\infty}, 0\right)$ for $v \lambda_{k}^{v}<0$.

(iv) $r \in\left(\lambda_{k}^{v} / f_{\infty}, 0\right)$ for $\lambda_{k}^{v}<0$.

Then problem (4) possesses two solutions $x_{k}^{+}$and $x_{k}^{-}$such that $x_{k}^{+}$has exactly $k-1$ zeros in $(0,1)$ and is positive near 0 and $x_{k}^{-}$has exactly $k-1$ zeros in $(0,1)$ and is negative near 0 . 
Proof. Define

$$
f^{[n]}(s):= \begin{cases}n s, & s \in\left[-\frac{1}{n}, \frac{1}{n}\right], \\ {\left[f\left(\frac{2}{n}\right)-1\right](n s-2)+f\left(\frac{2}{n}\right),} & s \in\left(\frac{1}{n}, \frac{2}{n}\right), \\ -\left[f\left(-\frac{2}{n}\right)+1\right](n s+2)+f\left(-\frac{2}{n}\right), & s \in\left(-\frac{2}{n},-\frac{1}{n}\right), \\ f(s), & s \in\left(-\infty,-\frac{2}{n}\right] \cup\left[\frac{2}{n},+\infty\right) .\end{cases}
$$

We consider the following problem:

$$
\begin{aligned}
& -x^{\prime \prime}=\alpha x^{+}+\beta x^{-}+\lambda r a(t) f^{[n]}(x), \quad 0<t<1, \\
& x(0)=x(1)=0 .
\end{aligned}
$$

Clearly, we can see that $\lim _{n \rightarrow+\infty} f^{[n]}(s)=f(s),\left(f^{[n]}\right)_{0}=$ $n$, and $\left(f^{[n]}\right)_{\infty}=f_{\infty}$.

Similar to the proof of [13, Theorem 4.1], by Lemma 8 and Remark 9, there exists an unbounded continuum $\mathscr{D}_{k}^{v[n]}$ of solutions of problem (45) emanating from $\left(\lambda_{k}^{v} / r n, 0\right)$, such that $\mathscr{D}_{k}^{v[n]} \subset\left(\Phi_{k}^{v} \cup\left\{\left(\lambda_{k}^{v} / r n, 0\right)\right\}\right)$, and $\mathscr{D}_{k}^{v[n]}$ joins $\left(\lambda_{k}^{v} / r n, 0\right)$ to $\left(\lambda_{k}^{v} / r f_{\infty}, \infty\right)$.

Taking $z_{n}=\left(\lambda_{k}^{v} / r n, 0\right)$ and $z^{*}=(0,0)$, we have that $z_{n} \rightarrow z^{*}$.

So condition (i) in Lemma 7 is satisfied with $z^{*}=(0,0)$.

Obviously

$$
r_{n}=\sup \left\{\lambda+\|x\| \mid(\lambda, x) \in \mathscr{D}_{k}^{\nu[n]}\right\}=\infty,
$$

and accordingly (ii) in Lemma 7 holds. (iii) in Lemma 7 can be deduced directly from the Arzela-Ascoli Theorem and the definition of $f^{[n]}$.

Therefore, by Lemma 7, $\lim \sup _{n \rightarrow \infty} \mathscr{D}_{k}^{v[n]}$ contains an unbounded connected component $\mathscr{D}_{k}^{v}$ with $(0,0) \in \mathscr{D}_{k}^{v}$.

Similar to Theorem 11, we obtain $\mathscr{D}_{k}^{v} \subset \mathcal{S}_{k}^{v}$.

Similar to the method of the proof of Case 2 of [13, Theorem 4.1], we can obtain that $\left(\lambda_{k}^{v} / r f_{\infty}, \infty\right) \in \mathscr{D}_{k}^{v}$.

Theorem 13. Let (A1), (A2), and (H4) hold. For $v \in\{+,-\}$, assume that one of the following conditions holds:

(i) $r \in(0,+\infty)$ for $\lambda_{k}^{\nu}>0$.

(ii) $r \in(0,+\infty) \cup(-\infty, 0)$ for $\nu \lambda_{k}^{v}>0$ or $\nu \lambda_{k}^{v}<0$.

(iii) $r \in(-\infty, 0)$ for $\lambda_{k}^{v}<0$.

Then problem (4) possesses two solutions $x_{k}^{+}$and $x_{k}^{-}$such that $x_{k}^{+}$has exactly $k-1$ zeros in $(0,1)$ and is positive near 0 and $x_{k}^{-}$has exactly $k-1$ zeros in $(0,1)$ and is negative near 0 .

Proof. Define

$$
f^{[n]}(s):= \begin{cases}\frac{1}{n} s, & s \in(-\infty,-2 n] \cup[2 n,+\infty) . \\ \frac{2+f(-n)}{n}(s+n)+f(-n), & s \in(-2 n,-n), \\ \frac{2-f(n)}{n}(s-n)+f(n), & s \in(n, 2 n), \\ -\left[f\left(-\frac{2}{n}\right)+1\right](n s+2)+f\left(-\frac{2}{n}\right), & s \in\left(-\frac{2}{n},-\frac{1}{n}\right), \\ {\left[f\left(\frac{2}{n}\right)-1\right](n s-2)+f\left(\frac{2}{n}\right),} & s \in\left(\frac{1}{n}, \frac{2}{n}\right), \\ n s, & s \in\left[-\frac{1}{n}, \frac{1}{n}\right] .\end{cases}
$$

We consider the following problem:

$$
\begin{aligned}
& -x^{\prime \prime}=\alpha x^{+}+\beta x^{-}+\lambda r a(t) f^{[n]}(x), \quad 0<t<1, \\
& x(0)=x(1)=0 .
\end{aligned}
$$

Clearly, we can see that $\lim _{n \rightarrow+\infty} f^{[n]}(s)=f(s),\left(f^{[n]}\right)_{0}=$ $n$, and $\left(f^{[n]}\right)_{\infty}=1 / n$.

Applying a similar method used in the proof of Theorem 12, we obtain an unbounded connected component $\mathscr{D}_{k}^{v} \subset \mathcal{S}_{k}^{v}$ with $(0,0) \in \mathscr{D}_{k}^{v}$. 
Similar to the method of the proof of Theorem 10, we can obtain that $(\infty, \infty) \in \mathscr{D}_{k}^{v}$. It follows that the result is obtained.

Theorem 14. Let (A1), (A2), and (H5) hold. For $v \in\{+,-\}$, assume that one of the following conditions holds:

(i) There exists $\lambda_{v k}^{v}>0$ for $\lambda_{k}^{v}>0$, such that $r \in$ $\left(\lambda_{v k}^{v},+\infty\right)$.

(ii) There exists $\nu \lambda_{v k}^{v}>0$ for $\nu \lambda_{k}^{v}>0$, such that $r \in$ $\left(-\infty, \lambda_{-k}^{-}\right) \cup\left(\lambda_{+k}^{+},+\infty\right)$. (iii) There exists $\nu \lambda_{v k}^{v}<0$ for $\nu \lambda_{k}^{v}<0$, such that $r \epsilon$ $\left(-\infty, \lambda_{+k}^{+}\right) \cup\left(\lambda_{-k}^{-},+\infty\right)$.

(iv) There exists $\lambda_{v k}^{v}<0$ for $\lambda_{k}^{v}<0$, such that $r \in$ $\left(-\infty, \lambda_{v k}^{v}\right)$

Then problem (4) possesses two solutions $x_{k}^{+}$and $x_{k}^{-}$such that $x_{k}^{+}$has exactly $k-1$ zeros in $(0,1)$ and is positive near 0 and $x_{k}^{-}$has exactly $k-1$ zeros in $(0,1)$ and is negative near 0 .

Proof. Define

$$
f^{[n]}(s):= \begin{cases}n s, & s \in(-\infty,-2 n] \cup[2 n,+\infty) . \\ \frac{2 n^{2}+f(-n)}{n}(s+n)+f(-n), & s \in(-2 n,-n), \\ f(s), & s \in(n, 2 n), \\ -\left[f\left(-\frac{2}{n}\right)+1\right](n s+2)+f\left(-\frac{2}{n}\right), & s \in\left(-\frac{2}{n},-\frac{1}{n}\right), \\ {\left[f\left(\frac{2}{n}\right)-1\right](n s-2)+f\left(\frac{2}{n}\right),} & s \in\left(\frac{1}{n}, \frac{2}{n}\right), \\ n s, & s \in\left[-\frac{1}{n}, \frac{1}{n}\right] .\end{cases}
$$

We consider the following problem:

$$
\begin{aligned}
& -x^{\prime \prime}=\alpha x^{+}+\beta x^{-}+\lambda r a(t) f^{[n]}(x), \quad 0<t<1, \\
& x(0)=x(1)=0 .
\end{aligned}
$$

Clearly, we can see that $\lim _{n \rightarrow+\infty} f^{[n]}(s)=f(s),\left(f^{[n]}\right)_{0}=$ $n$, and $\left(f^{[n]}\right)_{\infty}=n$.

Applying a similar method used in the proof of Theorem 12, we obtain an unbounded connected component $\mathscr{D}_{k}^{v} \subset \mathbb{R} \times \Sigma_{k}^{v}$ with $(0,0) \in \mathscr{D}_{k}^{v}$.

In the following, we can show that $(0, \infty) \in \mathscr{D}_{k}^{v}$. that

Assume that there exists a sequence $\left(\mu_{n}, x_{n}\right) \in \mathscr{D}_{k}^{v}$ such

$$
\begin{aligned}
\lim _{n \rightarrow \infty}\left(\left\|x_{n}\right\|+\left|\mu_{n}\right|\right) & =+\infty, \\
-x_{n}^{\prime \prime}=\alpha x_{n}^{+}+\beta x_{n}^{-}+\mu_{n} r a(t) & f\left(x_{n}\right), \\
& \\
x_{n}(0) & =x_{n}(1)=0 . t<1,
\end{aligned}
$$

We divide the proof into two steps.

Step 1. We show that $\lim _{n \rightarrow \infty}\left\|x_{n}\right\|=+\infty$.
If $\left\{x_{n}\right\}$ is bounded, then there exists a constant $M_{1}$ not depending on $n$ such that $\left\|x_{n}\right\| \leq M_{1}$. It follows that

$$
\lim _{n \rightarrow \infty}\left|\mu_{n}\right|=+\infty \text {. }
$$

Let

$$
\begin{aligned}
0 & =\tau(0, n)<\tau(1, n)<\tau(2, n)<\cdots<\tau(k-1, n) \\
& <\tau(k, n)=1
\end{aligned}
$$

denote the simple zeros of $x_{n}$ in $(0,1)$. Then, after choosing a subsequence and relabelling if necessary,

$$
\lim _{n \rightarrow+\infty} \tau(l, n)=\tau(l, \infty), \quad l \in\{0,1, \ldots, k\} .
$$

We claim that there exists $l_{0} \in\{0,1, \ldots, k\}$ such that

$$
\tau\left(l_{0}, \infty\right)<\tau\left(l_{0}+1, \infty\right) .
$$

Otherwise, we have

$$
\begin{aligned}
1 & =\sum_{l=0}^{k-1}(\tau(l+1, n)-\tau(l, n)) \\
& \longrightarrow \sum_{l=0}^{k-1}(\tau(l+1, \infty)-\tau(l, \infty))=0
\end{aligned}
$$

This is a contradiction. 
Let $[c, d] \subset\left(\tau\left(l_{0}, \infty\right), \tau\left(l_{0}+1, \infty\right)\right)$, with $c<d$. For all $n$ sufficiently large, we have $[c, d] \subset\left(\tau\left(l_{0}, n\right), \tau\left(l_{0}+1, n\right)\right)$. So $x_{n}(t)$ does not change its sign in $[c, d]$. Without loss of generality, we assume that $x_{n}(t)>0, t \in[c, d]$. Moreover, we have $\max _{t \in[c, d]}\left|x_{n}(t)\right| \leq M_{1}$. Combining this with the fact

$$
\frac{f\left(x_{n}\right)}{x_{n}(t)} \geq \inf \left\{\frac{f(s)}{s} \mid 0<s \leq M_{1}\right\}>0, \quad t \in[c, d]
$$

and with the relation

$$
-x_{n}^{\prime \prime}=\alpha x_{n}^{+}+\beta x_{n}^{-}+\mu_{n} r a(t) \frac{f\left(x_{n}\right)}{x_{n}(t)} x_{n}(t),
$$

$$
t \in[c, d] \text {. }
$$

Furthermore, we obtain that $\lim _{n \rightarrow+\infty} \mu_{n} r a(t)\left(f\left(x_{n}(t)\right) /\right.$ $\left.x_{n}(t)\right)= \pm \infty$ for any $t \in[c, d]$. Let $\varphi_{1}$ be the corresponding eigenfunction of $\lambda_{1}^{+}$of (1). If $\lim _{n \rightarrow+\infty} \mu_{n} r a(t)\left(f\left(x_{n}(t)\right) /\right.$ $\left.x_{n}(t)\right)=-\infty$ for any $t \in[c, d]$, applying Lemma 4 to $y_{n}$ and $\varphi_{1}$ on $[c, d]$, we can get that $\varphi_{1}$ must change its sign in $[c, d]$ for $n$ large enough. While, this is impossible. So we have that $\lim _{n \rightarrow+\infty} \mu_{n} r a(t)\left(f\left(x_{n}(t)\right) / x_{n}(t)\right)=+\infty$ for any $t \in[c, d]$. Applying Lemma 5 to $x_{n}(t)$ on $[c, d]$, we can get that $x_{n}(t)$ must change its sign in $[c, d]$ for $n$ large enough. This is a contradiction.

Step 2. We show that $\lim _{n \rightarrow \infty}\left|\mu_{n}\right|=0$.

Assume on the contrary that $\left|\mu_{n}\right|>c_{0}$. We consider the problem

$$
-x_{n}^{\prime \prime}=\alpha x_{n}(t)+\mu_{n} r a(t) \frac{f\left(x_{n}\right)}{x_{n}(t)} x_{n}(t), \quad t \in[c, d] .
$$

By Lemma 2.1 of [7], we have

$$
\lim _{n \rightarrow \infty} \min \left\{\left|x_{n}(t)\right| \mid t \in[c, d]\right\}=\infty .
$$

We have from (60) that we may assume, after taking a subsequence if necessary, that

$$
\begin{array}{ll}
\lim _{n \rightarrow \infty} \min \left\{x_{n}(t) \mid t \in[c, d]\right\}=+\infty & \left(x_{n}(t)>0\right) \text { or } \\
\lim _{n \rightarrow \infty} \max \left\{x_{n}(t) \mid t \in[c, d]\right\}=-\infty & \left(x_{n}(t)<0\right) .
\end{array}
$$

From $(A 1)$ and (61) and the condition $f_{\infty}=\infty$, we have that

$$
\lim _{n \rightarrow+\infty} \mu_{n} r a(t) \frac{f\left(x_{n}(t)\right)}{x_{n}(t)}= \pm \infty
$$

uniformly for $t \in[c, d]$.

Similar to the proof of Step 1, we can get a contradiction.

Theorem 15. Let (A1), (A2), and (H6) hold. For $v \in\{+,-\}$, assume that one of the following conditions holds:

(i) $r \in\left(\lambda_{k}^{v} / f_{\infty},+\infty\right)$ for $\lambda_{k}^{v}>0$.

(ii) $r \in\left(\lambda_{k}^{+} / f_{\infty},+\infty\right) \cup\left(-\infty, \lambda_{k}^{-} / f_{\infty}\right)$ for $\nu \lambda_{k}^{v}>0$.

(iii) $r \in\left(\lambda_{k}^{-} / f_{\infty},+\infty\right) \cup\left(-\infty, \lambda_{k}^{+} / f_{\infty}\right)$ for $\nu \lambda_{k}^{v}<0$.

(iv) $r \in\left(-\infty, \lambda_{k}^{v} / f_{\infty}\right)$ for $\lambda_{k}^{v}<0$.

Then problem (4) possesses two solutions $x_{k}^{+}$and $x_{k}^{-}$such that $x_{k}^{+}$has exactly $k-1$ zeros in $(0,1)$ and is positive near 0 and $x_{k}^{-}$has exactly $k-1$ zeros in $(0,1)$ and is negative near 0 .

Proof. Define

$$
f^{[n]}(s):= \begin{cases}\frac{1}{n} s, & s \in\left[-\frac{1}{n}, \frac{1}{n}\right], \\ {\left[f\left(\frac{2}{n}\right)-\frac{1}{n^{2}}\right](n s-2)+f\left(\frac{2}{n}\right),} & s \in\left(\frac{1}{n}, \frac{2}{n}\right), \\ -\left[f\left(-\frac{2}{n}\right)+\frac{1}{n^{2}}\right](n s+2)+f\left(-\frac{2}{n}\right), & s \in\left(-\frac{2}{n},-\frac{1}{n}\right), \\ f(s), & s \in\left(-\infty,-\frac{2}{n}\right] \cup\left[\frac{2}{n},+\infty\right) .\end{cases}
$$

We consider the following problem:

$$
\begin{aligned}
& -x^{\prime \prime}=\alpha x^{+}+\beta x^{-}+\lambda r a(t) f^{[n]}(x), \quad 0<t<1, \\
& x(0)=x(1)=0 .
\end{aligned}
$$

Clearly, we can see that $\lim _{n \rightarrow+\infty} f^{[n]}(s)=f(s),\left(f^{[n]}\right)_{0}=$ $1 / n$, and $\left(f^{[n]}\right)_{\infty}=f_{\infty}$.
Similar to the proof of [13, Theorem 4.1], by Lemma 8 and Remark 9, there exists an unbounded continuum $\mathscr{D}_{k}^{\nu[n]}$ of solutions of problem (64) emanating from $\left(n \lambda^{\nu} / r, 0\right)$, such that $\mathscr{D}_{k}^{v[n]} \subset\left(\Phi_{k}^{v} \cup\left\{\left(n \lambda^{\nu} / r, 0\right)\right\}\right)$, and $\mathscr{D}_{k}^{v[n]}$ joins $\left(n \lambda^{v} / r, 0\right)$ to $\left(\lambda^{\nu} / r f_{\infty}, \infty\right)$.

Taking $z_{n}=\left(n \lambda^{\nu} / r, 0\right)$ and $z^{*}=(\infty, 0)$, we have that $z_{n} \rightarrow z^{*}$.

So condition (i) in Lemma 7 is satisfied with $z^{*}=(\infty, 0)$.

Obviously

$$
r_{n}=\sup \left\{\lambda+\|x\| \mid(\lambda, x) \in \mathscr{D}_{k}^{v[n]}\right\}=\infty,
$$


and accordingly (ii) in Lemma 7 holds. (iii) in Lemma 7 can be deduced directly from the Arzela-Ascoli Theorem and the definition of $f^{[n]}$.

Therefore, by Lemma 7, lim $\sup _{n \rightarrow \infty} \mathscr{D}_{k}^{\nu[n]}$ contains an unbounded connected component $\mathscr{D}_{k}^{v}$ with $(\infty, 0) \in \mathscr{D}_{k}^{v}$.

Similar to Theorem 11, we obtain $\mathscr{D}_{k}^{v} \subset \mathcal{S}_{k}^{v}$.

Similar to the method of the proof of Case 2 of [13, Theorem 4.1], we can obtain that $\left(\lambda_{k}^{v} / r f_{\infty}, \infty\right) \in \mathscr{D}_{k}^{v}$.

Theorem 16. Let (A1), (A2), and (H7) hold. For $v \in\{+,-\}$, assume that one of the following conditions holds:

(i) There exists $\lambda_{v k}^{v}>0$ for $\lambda_{k}^{v}>0$, such that $r \in$ $\left(\lambda_{v k}^{v},+\infty\right)$.

$$
f^{[n]}(s):=\left\{\begin{array}{l}
\frac{1}{n} s, \\
\frac{2+f(-n)}{n}(s+n)+f(-n), \\
\frac{2-f(n)}{n}(s-n)+f(n), \\
-\left[f\left(-\frac{2}{n}\right)+\frac{1}{n^{2}}\right](n s+2)+f\left(-\frac{2}{n}\right), \\
{\left[f\left(\frac{2}{n}\right)-\frac{1}{n^{2}}\right](n s-2)+f\left(\frac{2}{n}\right),} \\
\frac{1}{n} s,
\end{array}\right.
$$

Proof. Define

$$
\begin{aligned}
& s \in(-\infty,-2 n] \cup[2 n,+\infty) . \\
& s \in(-2 n,-n), \\
& s \in(n, 2 n), \\
& s \in\left[-n,-\frac{2}{n}\right] \cup\left[\frac{2}{n}, n\right], \\
& s \in\left(-\frac{2}{n},-\frac{1}{n}\right), \\
& s \in\left(\frac{1}{n}, \frac{2}{n}\right), \\
& s \in\left[-\frac{1}{n}, \frac{1}{n}\right] .
\end{aligned}
$$

We consider the following problem:

$$
\begin{aligned}
& -x^{\prime \prime}=\alpha x^{+}+\beta x^{-}+\lambda r a(t) f^{[n]}(x), \quad 0<t<1, \\
& x(0)=x(1)=0 .
\end{aligned}
$$

Clearly, we can see that $\lim _{n \rightarrow+\infty} f^{[n]}(s)=f(s),\left(f^{[n]}\right)_{0}=$ $1 / n$, and $\left(f^{[n]}\right)_{\infty}=1 / n$.

Applying a similar method used in the proof of Theorem 15, we obtain an unbounded connected component $\mathscr{D}_{k}^{v} \subset \mathcal{S}_{k}^{v}$ with $(\infty, 0) \in \mathscr{D}_{k}^{v}$.

Similar to the proof of Theorem 10, we can show that $(\infty, \infty) \in \mathscr{D}_{k}^{v}$.
Theorem 17. Let (A1), (A2), and (H8) hold. For $v \in\{+,-\}$, assume that one of the following conditions holds:

(i) $r \in(0,+\infty)$ for $\lambda_{k}^{v}>0$.

(ii) $r \in(0,+\infty) \cup(-\infty, 0)$ for $\nu \lambda_{k}^{v}>0$ or $\nu \lambda_{k}^{v}<0$.

(iii) $r \in(-\infty, 0)$ for $\lambda_{k}^{v}<0$.

Then problem (4) possesses two solutions $x_{k}^{+}$and $x_{k}^{-}$such that $x_{k}^{+}$has exactly $k-1$ zeros in $(0,1)$ and is positive near 0 and $x_{k}^{-}$has exactly $k-1$ zeros in $(0,1)$ and is negative near 0 .

Proof. Define

$$
f^{[n]}(s):= \begin{cases}n s, & s \in(-\infty,-2 n] \cup[2 n,+\infty) . \\ \frac{2 n^{2}+f(-n)}{n}(s+n)+f(-n), & s \in(-2 n,-n), \\ \frac{2 n^{2}-f(n)}{n}(s-n)+f(n), & s \in(n, 2 n), \\ -\left[f\left(-\frac{2}{n}\right)+\frac{1}{n^{2}}\right](n s+2)+f\left(-\frac{2}{n}\right), & s \in\left(-\frac{2}{n},-\frac{1}{n}\right), \\ {\left[f\left(\frac{2}{n}\right)-\frac{1}{n^{2}}\right](n s-2)+f\left(\frac{2}{n}\right),} & s \in\left(\frac{1}{n}, \frac{2}{n}\right), \\ \frac{1}{n} s, & s \in\left[-\frac{1}{n}, \frac{1}{n}\right] .\end{cases}
$$


We consider the following problem:

$$
\begin{aligned}
& -x^{\prime \prime}=\alpha x^{+}+\beta x^{-}+\lambda r a(t) f^{[n]}(x), \quad 0<t<1, \\
& x(0)=x(1)=0 .
\end{aligned}
$$

Clearly, we can see that $\lim _{n \rightarrow+\infty} f^{[n]}(s)=f(s),\left(f^{[n]}\right)_{0}=$ $n$, and $\left(f^{[n]}\right)_{\infty}=1 / n$.

Applying a similar method used in the proof of Theorem 15, we obtain an unbounded connected component $\mathscr{D}_{k}^{v} \subset \mathcal{S}_{k}^{v}$ with $(\infty, 0) \in \mathscr{D}_{k}^{v}$.

Similar to the proof of Theorem 14, we can show that $(0, \infty) \in \mathscr{D}_{k}^{v}$.

Remark 18. Clearly, if $\alpha=\beta=0$, [6, 7] studied the problem (4) under conditions (A2) and (H4) or (H8). Hence, the results of Theorems 13 and 17 improve on Theorems 1.1 and 1.2 of [6] or [7].

Remark 19. Note that if $\alpha=\beta \equiv 0$ the result of Theorems 1017 is equivalent to those of Theorems 2.1-2.8 of [10] (when $p=2$ ), respectively. Hence, Theorems 10-17 extend the corresponding results of [10] (when $p=2$ ).

Remark 20. The nonlinear term of (4) is not necessarily homogeneous linearizable at the origin and infinity because of the influence of the term $\alpha x^{+}+\beta x^{-}$. Clearly, so the bifurcation results of $[1,2,8-12]$ cannot be applied directly to obtain our results.

Remark 21. We consider the cases of $f_{0}, f_{\infty} \notin(0, \infty)$, while the authors of [13] only studied the cases of $f_{0}, f_{\infty} \in(0, \infty)$. Hence, Theorems 10-17 extend Theorem 4.1 of [13].

Remark 22. Previous cases have been investigated by several authors by applying also different methods. In particular, under the conditions $\alpha=\beta=0$ and (H4) or (H8), we can see [24-29].

\section{Conflict of Interests}

The author declares that there is no conflict of interests regarding the publication of this paper.

\section{Acknowledgments}

Thanks are given to the anonymous referee for his/her valuable suggestions. The authors were supported by the NSFC (no. 11561038) and Gansu Provincial National Science Foundation of China (no. 145RJZA087).

\section{References}

[1] P. H. Rabinowitz, "Some global results for nonlinear eigenvalue problems," Journal of Functional Analysis, vol. 7, no. 3, pp. 487513, 1971.

[2] P. H. Rabinowitz, "Some aspects of nonlinear eigenvalue problems," Rocky Mountain Journal of Mathematics, vol. 3, no. 2, pp. 161-202, 1973.
[3] B. H. Im, E. K. Lee, and Y.-H. Lee, "A global bifurcation phenomenon for second order singular boundary value problems," Journal of Mathematical Analysis and Applications, vol. 308, no. 1, pp. 61-78, 2005.

[4] R. Ma and B. Thompson, "Nodal solutions for nonlinear eigenvalue problems," Nonlinear Analysis, Theory, Methods and Applications, vol. 59, no. 5, pp. 707-718, 2004.

[5] B. P. Rynne, "Global bifurcation for 2 mth-order boundary value problems and infinitely many solutions of superlinear problems," Journal of Differential Equations, vol. 188, no. 2, pp. 461-472, 2003.

[6] R. Ma and B. Thompson, "Multiplicity results for secondorder two-point boundary value problems with superlinear or sublinear nonlinearities," Journal of Mathematical Analysis and Applications, vol. 303, no. 2, pp. 726-735, 2005.

[7] R. Ma, "Nodal solutions of second-order boundary value problems with superlinear or sublinear nonlinearities," Nonlinear Analysis, Theory, Methods and Applications, vol. 66, no. 4, pp. 950-961, 2007.

[8] G. Dai and R. Ma, "Unilateral global bifurcation phenomena and nodal solutions for $p$-Laplacian," Journal of Differential Equations, vol. 252, no. 3, pp. 2448-2468, 2012.

[9] E. N. Dancer, "On the structure of solutions of non-linear eigenvalue problems," Indiana University Mathematics Journal, vol. 23, pp. 1069-1076, 1974.

[10] G. Dai, "Bifurcation and nodal solutions for p-Laplacian problems with non-asymptotic nonlinearity at 0 or $\infty$," Applied Mathematics Letters, vol. 26, no. 1, pp. 46-50, 2013.

[11] E. N. Dancer, "Bifurcation from simple eigenvalues and eigenvalues of geometric multiplicity one," Bulletin of the London Mathematical Society, vol. 34, no. 5, pp. 533-538, 2002.

[12] J. López-Gómez, Spectral Theory and Nonlinear Functional Analysis, Chapman \& Hall, CRC Press, Boca Raton, Fla, USA, 2001.

[13] R. Ma and G. Dai, "Global bifurcation and nodal solutions for a Sturm-Liouville problem with a nonsmooth nonlinearity," Journal of Functional Analysis, vol. 265, no. 8, pp. 1443-1459, 2013.

[14] H. Berestycki, "On some nonlinear Sturm-Liouville problems," Journal of Differential Equations, vol. 26, no. 3, pp. 375-390, 1977.

[15] K. Schmitt and H. L. Smith, "On eigenvalue problems for nondifferentiable mappings," Journal of Differential Equations, vol. 33, no. 3, pp. 294-319, 1979.

[16] G. Dai and R. Ma, "Unilateral global bifurcation for $p$-Laplacian with non- $p$-1-linearization nonlinearity," Discrete and Continuous Dynamical Systems Series: A, vol. 35, no. 1, pp. 99-116, 2015.

[17] G. Dai and R. Ma, "Global bifurcation, Berestycki's conjecture and one-sign solutions for p-Laplacian," Nonlinear Analysis, Theory, Methods and Applications, vol. 91, pp. 51-59, 2013.

[18] B. P. Rynne, "p-Laplacian problems with jumping nonlinearities," Journal of Differential Equations, vol. 226, no. 2, pp. 501$524,2006$.

[19] M. Picone, "Sui valori eccezionali di un parametro da cui dipende un'equazione differenziale lineare ordinaria del second ordine," Annali della Scuola Normale Superiore di Pisa, vol. 11, pp. 1-141, 1910.

[20] T. Kusano, J. Jaroš, and N. Yoshida, "A Picone-type identity and Sturmian comparison and oscillation theorems for a class of half-linear partial differential equations of second order," Nonlinear Analysis, Theory, Methods and Applications, vol. 40, pp. 381-395, 2000. 
[21] G. T. Whyburn, Topological Analysis, Princeton University Press, Princeton, NJ, USA, 1958.

[22] R. Ma and Y. An, "Global structure of positive for superlinear second-order m-point boundary value problems," Topological Methods in Nonlinear Analysis, vol. 34, no. 2, pp. 279-290, 2009.

[23] A. Ambrosetti, R. M. Calahorrano, and F. R. Dobarro, "Global branching for discontinuous problems," Commentationes Mathematicae Universitatis Carolinae, vol. 31, no. 2, pp. 213-222, 1990.

[24] L. H. Erbe and H. Wang, "On the existence of positive solutions of ordinary differential equations," Proceedings of the American Mathematical Society, vol. 120, no. 3, pp. 743-743, 1994.

[25] A. Capietto and W. Dambrosio, "Boundary value problems with sublinear conditions near zero," Nonlinear Differential Equations and Applications, vol. 6, no. 2, pp. 149-172, 1999.

[26] N. Hirano, "Existence of infinitely many solutions for sublinear elliptic problems," Journal of Mathematical Analysis and Applications, vol. 278, no. 1, pp. 83-92, 2003.

[27] Y. Naito and S. Tanaka, "On the existence of multiple solutions of the boundary value problem for nonlinear second-order differential equations," Nonlinear Analysis, Theory, Methods and Applications, vol. 56, no. 6, pp. 919-935, 2004.

[28] J. Shi and M. Yao, "Positive solutions for elliptic equations with singular nonlinearity," Electronic Journal of Differential Equations, vol. 4, pp. 1-11, 2005.

[29] G. Bonanno and A. Sciammetta, "Existence and multiplicity results to Neumann problems for elliptic equations involving the p-Laplacian," Journal of Mathematical Analysis and Applications, vol. 390, no. 1, pp. 59-67, 2012. 


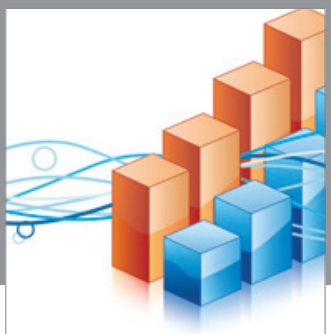

Advances in

Operations Research

vatem alat4

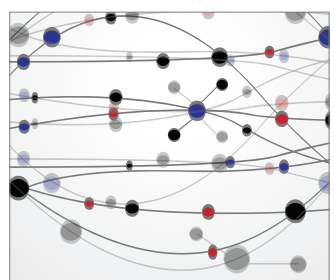

\section{The Scientific} World Journal
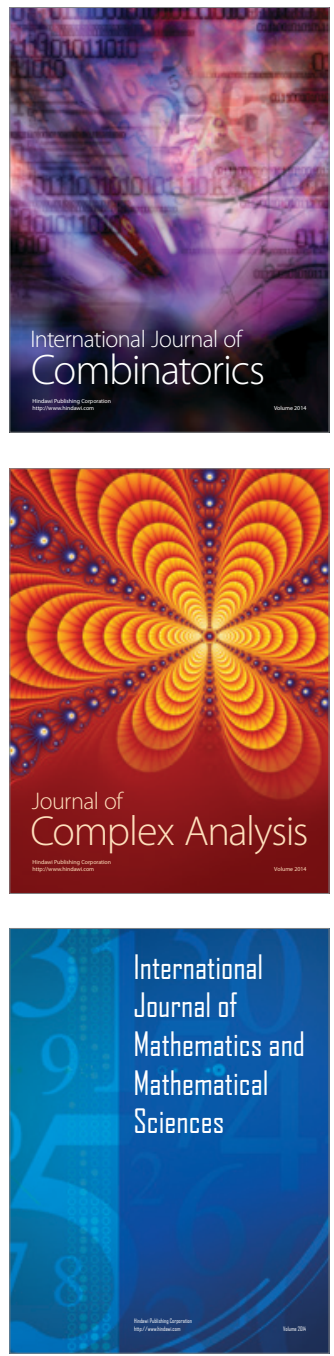
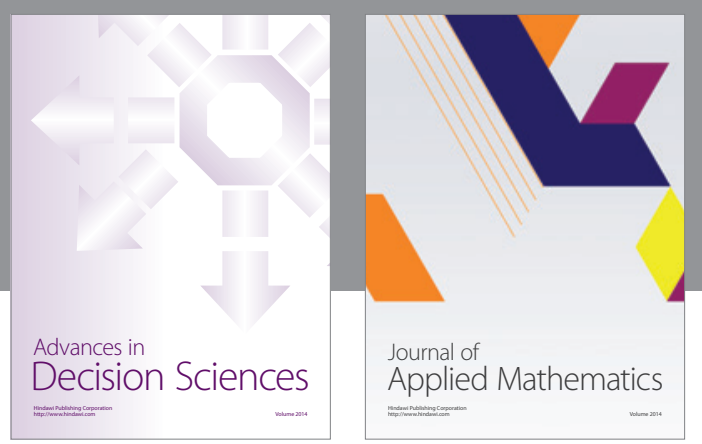

Algebra

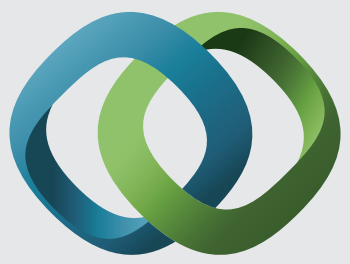

\section{Hindawi}

Submit your manuscripts at

http://www.hindawi.com
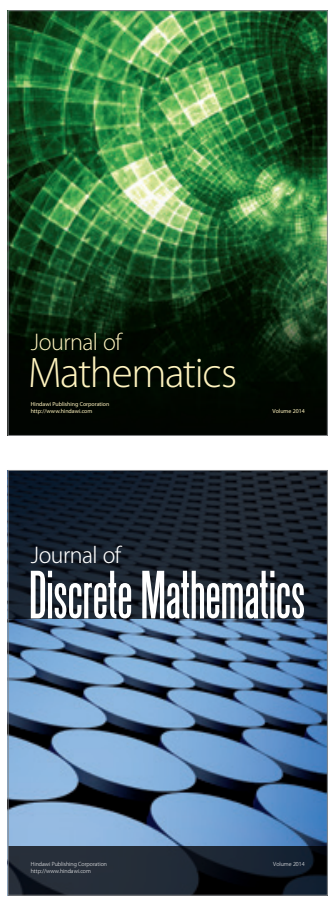

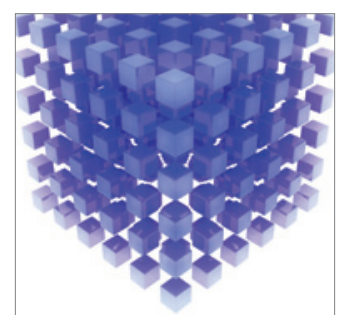

Mathematical Problems in Engineering
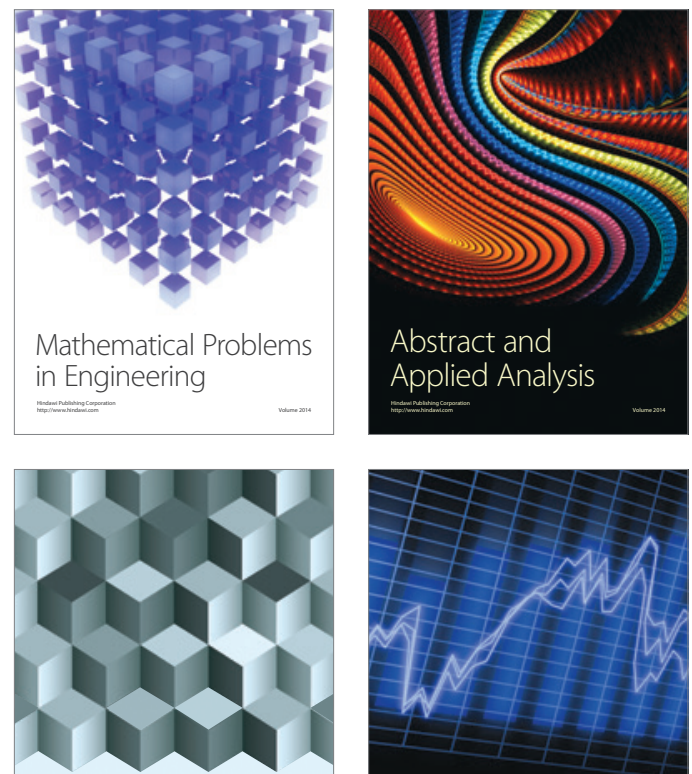

Journal of

Function Spaces

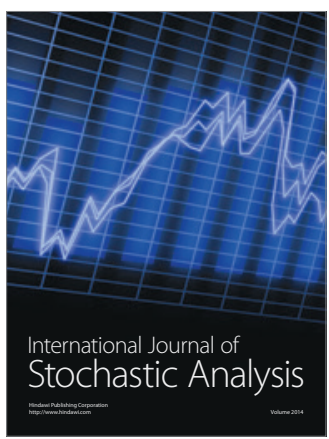

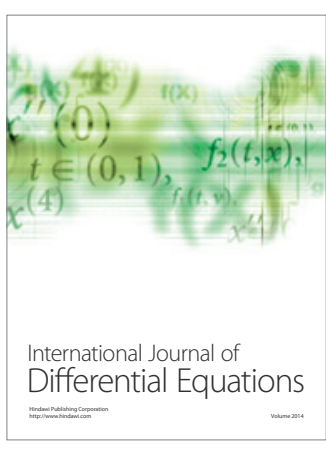
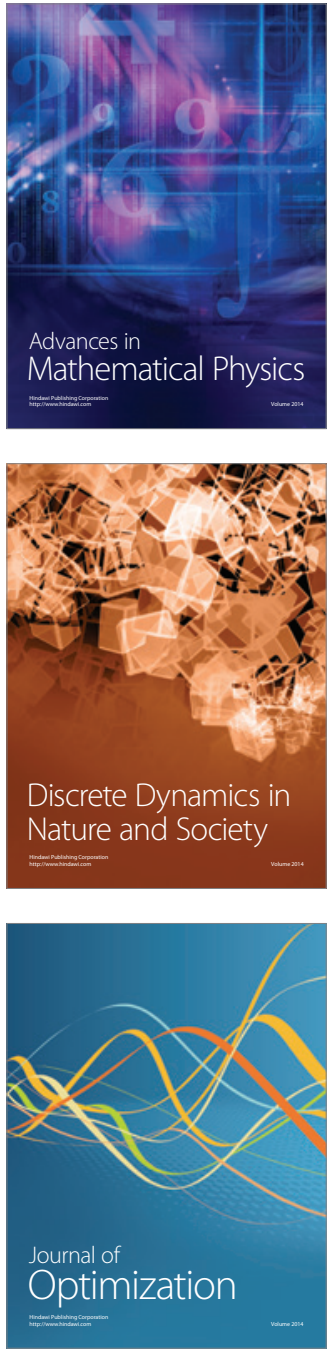